\title{
Sinterização e propriedades mecânicas do compósito $\mathrm{Y}-\mathrm{TZP} / \mathrm{Al}_{2} \mathrm{O}_{3}$
}

\section{(Sintering and mechanical properties of the $\mathrm{Y}-\mathrm{TZP} / \mathrm{Al}_{2} \mathrm{O}_{3}$ composites)}

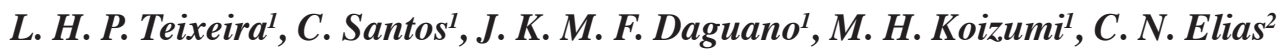 \\ ${ }^{1}$ Escola de Engenharia de Lorena - EEL, Universidade de S. Paulo - USP \\ Pólo Urbo-Industrial, Gleba AI-6 s/n, Lorena, SP 12600-000 \\ ${ }^{2} I M E$ - Instituto Militar de Engenharia, Pça. General Tibúrcio 80, Praia Vermelha, Rio de Janeiro, RJ 22290-270 \\ claudinei@demar.eel.usp.br
}

\begin{abstract}
Resumo
Foi estudado o efeito da adição de $\mathrm{Al}_{2} \mathrm{O}_{3}$ nas propriedades de cerâmicas à base de $\mathrm{ZrO}_{2}$ tetragonal estabilizada com ítria (Y-TZP) visando sua aplicação como material dentário. Amostras contendo $0,10,20$ e $30 \%$ em peso de $\mathrm{Al}_{2} \mathrm{O}_{3}$ foram compactadas por prensagem uniaxial a frio (80 MPa) e em seguida sinterizadas ao ar a 1500,1550 e $1600{ }^{\circ} \mathrm{C}$ por 120 min. Os efeitos destas condições de sinterização nas propriedades dos materiais foram analisados por difração de raios X e microscopia eletrônica de varredura. As propriedades mecânicas de dureza e tenacidade à fratura foram determinadas, utilizando-se o método de indentação Vickers. Além disso, algumas amostras foram submetidas a ensaio de flexão em 4 pontos. Cerâmicas de elevada densidade relativa foram obtidas, com dureza variando entre 13,4 e $15,8 \mathrm{GPa}$, dependendo da quantidade de $\mathrm{Al}_{2} \mathrm{O}_{3}$ adicionada à matriz de $\mathrm{ZrO}$. Por outro lado, não foi verificada uma influencia significativa da adição de $\mathrm{Al}_{2} \mathrm{O}_{3}$ na tenacidade à fratura, que apresentou valores próximos de $8 \mathrm{MPa} \cdot \mathrm{m}^{1 / 2}$, em quase todas as situações. A boa dureza, a elevada tenacidade à fratura, a boa resistência à fratura $\left(\sigma_{\mathrm{f}}=685 \mathrm{MPa}\right)$ e o módulo de Weibull $(\mathrm{m}=11)$ apresentados pelos materiais com adições de $20 \%$ de $\mathrm{Al}_{2} \mathrm{O}_{3}$, indicam a confiabilidade do compósito como um potencial biomaterial para uso na implantodontia.
\end{abstract}

Palavras-chave: $\mathrm{ZrO}_{2}, \mathrm{Al}_{2} \mathrm{O}_{3}$, sinterização, propriedades mecânicas.

\begin{abstract}
In this work, the influence of $\mathrm{Al}_{2} \mathrm{O}_{3}$ addition on the tetragonal $\mathrm{ZrO}$, ceramic properties was studied, aiming the use of this ceramic as dental materials. Samples containing 0, 10, 20 e $30 \mathrm{wt} \%$ were cold pressed $(80 \mathrm{MPa})$, and sintered in air, at 1500, 1550 e $1600^{\circ} \mathrm{C}$, for $120 \mathrm{~min}$. The effects of the sintering conditions were analyzed by X-ray diffraction and Scanning Electron Microscopy. Hardness and fracture toughness were determined by Vicker's indentation method. Furthermore, samples were submitted to the 4 point bending test. Dense ceramics were obtained, with hardness values varying around 13.4 and $15.8 \mathrm{GPa}$, depending on the $\mathrm{Al}_{2} \mathrm{O}_{3}$ content added in the $\mathrm{ZrO}_{2}$ matrix. On the other hand, no significant difference in the fracture toughness was detected by the $\mathrm{Al}_{2} \mathrm{O}_{3}$ addition, with $\mathrm{K}_{\mathrm{IC}}$ around $8 \mathrm{MPa} . \mathrm{m}^{1 / 2}$, in all conditions. The high hardness, fracture toughness, flexural strength $\left(\sigma_{f}=685 \mathrm{MPa}\right)$ and reliability $(\mathrm{m}=11)$ presented by the ceramic composites with $20 \mathrm{wt} . \% \mathrm{Al}_{2} \mathrm{O}_{3}$, indicates the potential of these materials as bioceramics for dental materials.
\end{abstract}

Keywords: $\mathrm{ZrO}_{2}, \mathrm{Al}_{2} \mathrm{O}_{3}$, sintering, mechanical properties.

\section{INTRODUÇÃO}

O desenvolvimento de tecnologias para a produção de novos materiais biocompátiveis tem sido motivado pela demanda de materiais que executem novas funções ou desempenhem antigas funções de forma mais adequada [12]. A elaboração de materiais cerâmicos avançados para esse fim, teve início na década de 70 e tem alcançado resultados promissores, sendo inclusive verificada uma contínua evolução no desenvolvimento e uso destes materiais em diversas aplicações.

Uma grande evolução nas técnicas de restauração dentária vem sendo estabelecida pelo uso de materiais cerâmicos. Esses materiais apresentam vantagens relativas devidas ao ótimo desempenho das suas propriedades funcionais, principalmente estética, biocompatibilidade e estabilidade química [1-3]. A tendência das técnicas de cerâmica dental vem sendo a eliminação da subestrutura metálica das restaurações, inclusive das restaurações sobre implantes, visando uma melhor estética e utilizando para isso, cerâmicas de maior tenacidade à fratura, minimizando a sua fragilidade.

A utilização de cerâmicas a base de alumina $\left(\mathrm{Al}_{2} \mathrm{O}_{3}\right)$ e zircônia $\left(\mathrm{ZrO}_{2}\right)$ de alta densidade relativa vem sendo proposta, em função de a alumina ter apresentado uma excelente biocompatibilidade, alta dureza e resistência ao desgaste, embora, tenha exibido moderada resistência à flexão e tenacidade [4-5]. A zircônia pura não pode ser utilizada na fabricação de peças sem a adição de estabilizantes. A zircônia estabilizada com ítria (Y-TZP) se 
tornou uma alternativa popular a alumina, como cerâmica estrutural, uma vez que apresenta maior resistência à flexão, maior tenacidade à fratura e menor módulo de elasticidade [5-10]. Além de suas propriedades mecânicas, a zircônia se torna esteticamente bastante interessante quando polida.

Diante das evidências do papel da zircônia como agente tenacificador de cerâmicas, vários sistemas matriz cerâmicazircônia têm sido estudados. Tal reforço é conseqüência da transformação induzida por tensão da fase tetragonal para fase monoclínica das partículas de zircônia, a qual é acompanhada de uma expansão volumétrica (3 a 6\%) [5]. A transformação absorve parte da energia necessária para a propagação da trinca, gerando um aumento da tenacidade à fratura.

Dois tipos de compósitos podem ser preparados a partir do sistema: uma matriz de zircônia estabilizada com ítria reforçada com partículas de alumina (ATZ) ou uma matriz de alumina reforçada com partículas de zircônia (ZTA). Com ambos materiais bifásicos (ZTA e ATZ), é esperada a obtenção de maiores valores de tenacidade à fratura quando comparado com os materiais cerâmicos monofásicos [4, 11-14].

$\mathrm{O}$ objetivo desse trabalho foi verificar a influência da temperatura de sinterização e da fração de alumina na densificação e nas propriedades mecânicas dos compósitos $\mathrm{ZrO}_{2}-\mathrm{Al}_{2} \mathrm{O}_{3}$.

\section{EXPERIMENTAL}

\section{Processamento}

Pós de $\mathrm{ZrO}_{2}$ estabilizado com $3 \%$ mol de ítria, com tamanho médio de partículas de $0,85 \mu \mathrm{m}$, apresentando $20 \%$ de fase monoclínica residual, TZ-3YSB (Tosoh, Japão) e $\mathrm{Al}_{2} \mathrm{O}_{3}$ (SG-1000 - Almatis, grupo Alcoa), com tamanho médio de partículas de $0,45 \mu \mathrm{m}$, foram usados como materiais iniciais. $\mathrm{O}$ pó de $\mathrm{ZrO}_{2}$ obtido pela técnica spray-dry possui ligante em sua composição para facilitar a compactação.

Foram preparadas quatro composições com mistura dos óxidos variando o percentual de adição de alumina na zircônia, em 0, 10, 20 e 30\% em peso, nas misturas. As misturas de pós foram preparadas via úmido, em moinho atritor por $4 \mathrm{~h}$ usando álcool isopropílico como meio e utilizando bolas de $\mathrm{ZrO}_{2}$ sinterizada com diâmetro médio de $2 \mathrm{~mm}$. Após a moagem, todas as misturas apresentaram tamanho médio de partículas de $0,68 \mu \mathrm{m}$. Essas misturas de pós foram secadas em estufa a $90{ }^{\circ} \mathrm{C}$ por $24 \mathrm{~h}$, e em seguida, desaglomeradas e prensadas uniaxialmente a frio sob pressão de $80 \mathrm{MPa}$.

Amostras com $20 \mathrm{~mm}$ de diâmetro e $8 \mathrm{~mm}$ de altura foram compactadas e sinterizadas em temperaturas de 1500 , 1550 e $1600{ }^{\circ} \mathrm{C}$. As taxas de aquecimento variaram em função da temperatura alcançada, que foram $10^{\circ} \mathrm{C} / \mathrm{min}$ até $1100^{\circ} \mathrm{C}, 5^{\circ} \mathrm{C} / \mathrm{min}$ até $1400^{\circ} \mathrm{C}$ e $3^{\circ} \mathrm{C} / \mathrm{min}$ até a temperatura final. A taxa de resfriamento foi de $5^{\circ} \mathrm{C} / \min$ até $1400{ }^{\circ} \mathrm{C}$ e de $3{ }^{\circ} \mathrm{C} / \mathrm{min}$ até $1100^{\circ} \mathrm{C}$, com o resfriamento feito de forma automática pelo forno. $\mathrm{O}$ tempo de patamar de sinterização foi constante e igual a $120 \mathrm{~min}$, para todas as temperaturas.

\section{Caracterizações}

As misturas de pós foram caracterizadas quanto à sua distribuição de tamanho de partícula utilizando o método de sedimentação gravimétrica e os compactos foram caracterizados quanto à sua densidade relativa à verde.

As amostras sinterizadas foram caracterizadas quanto a sua retração linear e perda de massa, através das medições antes e após a sinterização. O cálculo da massa específica das amostras sinterizadas foi realizado, aplicando-se o método de imersão utilizando o princípio de Arquimedes.

As fases presentes nas amostras sinterizadas foram identificadas por difração de raios $\mathrm{X}$, utilizando radiação $\mathrm{Cu}-\mathrm{K}_{\alpha}$ com varredura entre $10^{\circ}$ e $80^{\circ}$, com passo de $0,05^{\circ} \mathrm{e}$ velocidade de $2 \mathrm{~s} /$ ponto de contagem. A fração monoclínica $\mathrm{X}_{\mathrm{m}}$ foi calculada pelo método de Garvie e Nicholson [15]. A fração volumétrica $V_{m}$ foi determinada segundo a ref [16].

As amostras sinterizadas foram analisadas quanto a sua microestrutura, através de microscopia eletrônica de varredura, visando obter o tamanho médio de grãos da $\mathrm{ZrO}_{2}$ e $\mathrm{Al}_{2} \mathrm{O}_{3}$, em função da composição da amostra e da temperatura de sinterização empregada. As amostras foram lixadas, polidas e sofreram ataque térmico a $1300{ }^{\circ} \mathrm{C}$, por 15 min, para revelação dos contornos de grão, e analisada utilizando analisador de imagem.

\section{Propriedades mecânicas}

As propriedades mecânicas de dureza e tenacidade à fratura foram determinadas utilizando-se o método de indentação Vickers. Por razões estatísticas, foram realizadas 21 impressões Vickers nas superfícies de cada uma das amostras polidas, usando-se uma carga de 2000 gf (carga máxima do indentador) aplicada por $30 \mathrm{~s}$. A tenacidade à fratura foi calculada através da equação proposta [17] para trincas do tipo Palmqvist, com relação entre o comprimento da trinca e a diagonal de impressão $(c / a)<3,5$. Para realização dessas medidas foram utilizados valores de módulo de elasticidade dos materiais monolíticos $\mathrm{E}_{\mathrm{ZrO} 2}=190 \mathrm{GPa}$ e $\mathrm{E}_{\mathrm{Al} 203}=400 \mathrm{GPa}$, aplicando-se a regra das misturas.

Para realização dos testes de flexão em 4 pontos, um lote com 21 amostras da composição 80:20 sinterizadas a 1600 ${ }^{\circ} \mathrm{C}$ foram retificadas, lixadas e polidas, obtendo-se barras de $4 \mathrm{~mm} \times 3 \mathrm{~mm} \times 45 \mathrm{~mm}$, de acordo com a norma ASTM C1116-94. Os testes foram conduzidos usando ensaio de flexão em 4 pontos, com espaçamento entre roletes de 40 $\mathrm{mm}\left(\mathrm{I}_{1}\right)$ e $20 \mathrm{~mm}\left(\mathrm{I}_{2}\right)$. A velocidade de carregamento foi de $0,5 \mathrm{~mm} / \mathrm{s}$. A resistência à flexão das amostras foi calculada pela equação (A):

$$
\sigma_{\mathrm{f}}=\frac{3}{2} \mathrm{~F}_{\mathrm{A}} \times \frac{\left(\mathrm{I}_{1}-\mathrm{I}_{2}\right)}{\mathrm{b} \times \mathrm{h}^{2}}
$$

$\sigma_{\mathrm{f}}$ - Resistência a flexão (à fratura) (MPa), $\mathrm{F}_{\mathrm{a}}$ - Carga de ruptura $(\mathrm{N}), \mathrm{b}$ - Largura da amostra $(\mathrm{mm}), \mathrm{h}$ - Altura da amostra $(\mathrm{mm}), \mathrm{I}_{1}$ - Maior espaço entre roletes $(\mathrm{mm}), \mathrm{I}_{2}$ Menor espaço entre roletes $(\mathrm{mm})$ 
Para a avaliação estatística da resistência a fratura a probabilidade de falha obtida pela distribuicão de Weibull [18] foi realizada utilizando a equação (B):

$$
\mathrm{P}=1-\exp \left\{\left[-\frac{\sigma}{\sigma_{0}}\right]^{\mathrm{m}}\right\}
$$

P - Probabilidade de Falha, $\mathrm{m}$ - Módulo de Weibull, $\sigma_{0}$ - Resistência Característica e $\sigma$ - Resistência à fratura por flexão.

Os parâmetros de Weibull $m$ e $\sigma_{0}$, são obtidos transformando a Equação (B) na Equação (C) e plotando $l n$ $\ln [1 /(1-P)]$ vs $\ln \sigma$.

$$
\ln \ln \frac{1}{1-\mathrm{P}}=\mathrm{m} \cdot \ln \sigma-\mathrm{m} \cdot \ln \sigma_{0}
$$

O valor da tensão para $63 \%$ de probabilidade de falha foi estimado como parâmetro de referência e o modulo de Weibull foi determinado usando um fator de correção de 0,938 para 21 amostras, de acordo com a norma alemã DIN-51-110 [19].

\section{RESULTADOS E DISCUSSÃO}

\section{Sinterização}

A Fig. 1 apresenta os resultados de densidade relativa em função da temperatura de sinterização e do teor de $\mathrm{Al}_{2} \mathrm{O}_{3}$.

Foi observado que há um leve acréscimo de densificação, em função do aumento da temperatura de sinterização, em todos os casos. Fato interessante é que acima de $1500{ }^{\circ} \mathrm{C}$ todos os materiais estudados apresentaram densidade relativa acima de $99 \%$, o que favorece as propriedades mecânicas dos materiais, eleva sua confiabilidade e resultam em produtos de melhor comportamento em aplicações estruturais. Notouse que, de uma forma geral, os compósitos apresentaram níveis de porosidade bastante reduzidos, e próximos, independentes dos teores de $\mathrm{Al}_{2} \mathrm{O}_{3}$ utilizados. Dessa forma, os teores de $\mathrm{Al}_{2} \mathrm{O}_{3}$ não influenciaram nos níveis finais de densificação. Isso é justificável, pois as misturas de pós apresentaram distribuição de tamanho de partículas bastante próximas. Além disso, os resultados de densidade relativa à verde não variaram em função da adição de $\mathrm{Al}_{2} \mathrm{O}_{3}$, tendo, em média, densidade em torno de $50 \%$.

A Fig. 2 Apresenta os resultados de perda percentual de massa e da retração linear das amostras sinterizadas em função do teor de alumina nas amostras.

Pode-se observar que a perda de massa foi muito pequena para todos os casos analisados, não tendo grande variação em função da mudança da temperatura de sinterização e da quantidade de alumina adicionada. Essa perda de massa pode ser atribuída a volatização de compostos orgânicos utilizados no processo de fabricação e de compactação (ligantes e ou lubrificantes).

Considerando que as amostras foram compactadas e sinterizadas seguindo a mesma seqüência, foi observado que quanto maior o teor de alumina, uma leve redução dos níveis de retração é observada, seguindo uma mesma tendência para as diferentes temperaturas.
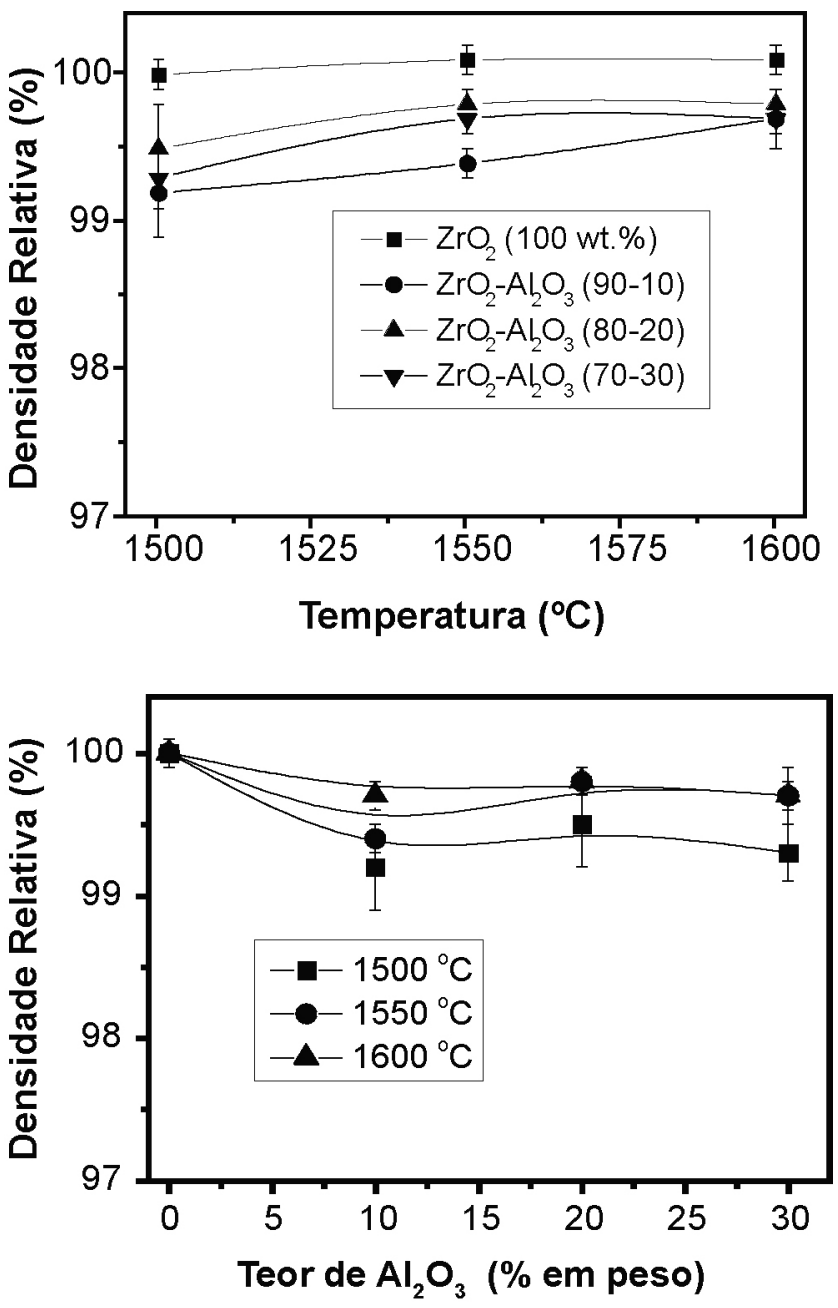

Figura 1: Influência da temperatura de sinterização e do teor de alumina na densificação das amostras.

[Figure 1: Influence of the sintering time and $\mathrm{Al}_{2} \mathrm{O}_{3}$ content in the densification of the samples.]

A Fig. 3 apresenta difratogramas para as diferentes amostras sinterizadas a $1600{ }^{\circ} \mathrm{C}$. As amostras sinterizadas a 1500 e $1550{ }^{\circ} \mathrm{C}$ apresentaram difratogramas similares aos das amostras sinterizadas a $1600{ }^{\circ} \mathrm{C}$.

É observada, para as diferentes composições, somente a presença de zircônia estabilizada tetragonal, indicando que o percentual de zircônia monoclínica presente no pó de partida foi integralmente transformada. Nota-se ainda que nas composições com adições de alumina, que há também ausência da fase $\mathrm{ZrO}_{2}$ monoclínica, indicando também total transformação durante a sinterização. Isso indica que a alumina não influenciou nas taxas de transformação de fase da zircônia, durante a sinterização. Nota-se ainda, um aumento da intensidade dos picos da fase $\alpha-\mathrm{Al}_{2} \mathrm{O}_{3}$, consistente com o aumento do teor desse material na composição final do compósito.

É conhecido [5] que a aplicação de tensões sobre a superfície da zircônia tetragonal pode gerar transformações T-M (tetragonal-monoclínica), como no caso de aplicação de esforços de lixamento e polimento. A Fig. 4 apresenta difratogramas relativos às superfícies após preparação superficial (lixamento e subseqüente polimento), de 

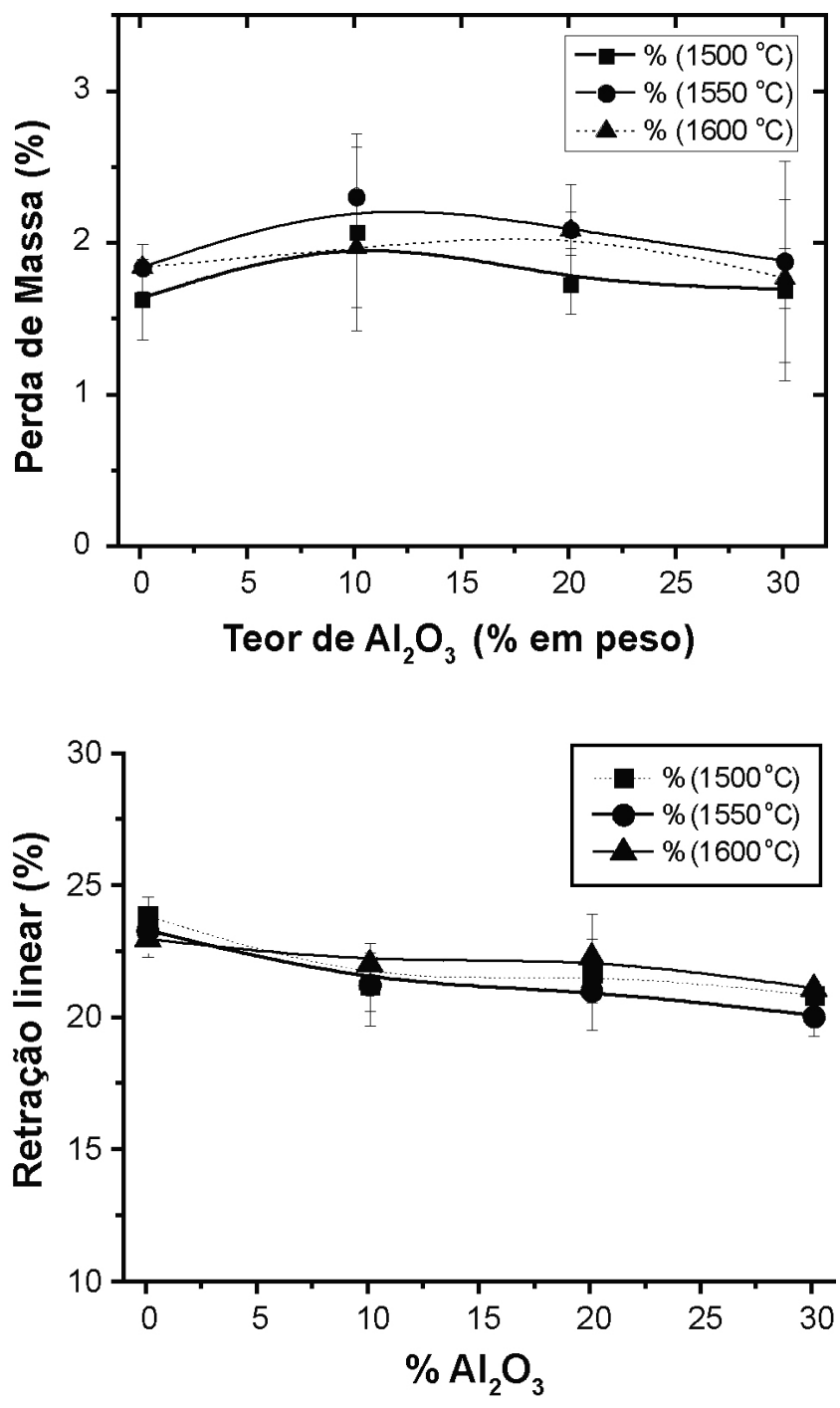

Figura 2: Influência do teor de alumina na perda de massa e na retração linear das amostras sinterizadas.

[Figure 2: Influence of the $\mathrm{Al}_{2} \mathrm{O}_{3}$ content and sintering temperature on the weight loss and shrinkage of the sintered samples.]

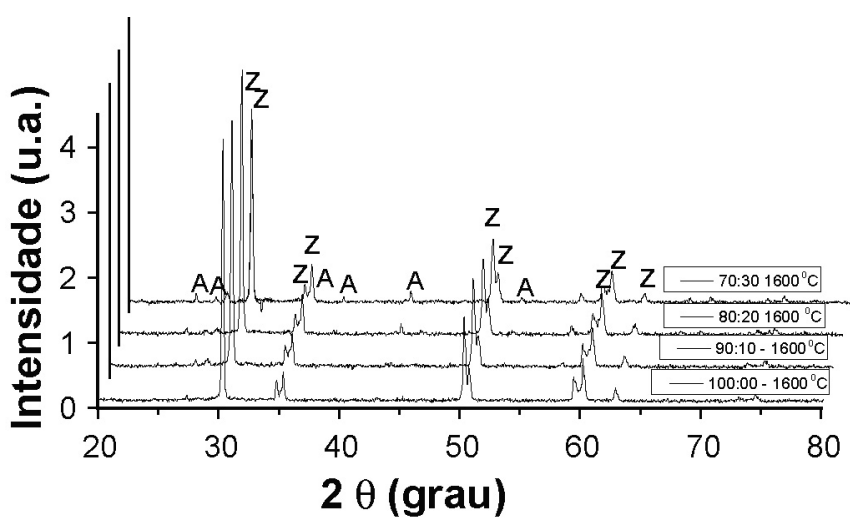

Figura 3: Difratogramas de raios $\mathrm{X}$ dos compósitos $\mathrm{ZrO}_{2}: \mathrm{Al}_{2} \mathrm{O}_{3}$ sinterizados a $1600{ }^{\circ} \mathrm{C}$.

[Figure 3: X-Ray diffraction patterns of the $\mathrm{ZrO}_{2}: \mathrm{Al}_{2} \mathrm{O}_{3}$ composites, sintered at $\left.1600^{\circ} \mathrm{C}\right]$ amostras sinterizadas a 1500 e $1600{ }^{\circ} \mathrm{C}-120$ min, visando detectar tal transformação. Dentro do limite de detecção do difratômetro (cerca de $2 \%$ ), não é notada a presença da fase $\mathrm{ZrO}$ monoclínica caracterizada por picos de difração nas posições $2 \theta=28^{\circ}$ e $2 \theta=31^{\circ}$. Assim pode-se inferir que o teor de transformação T-M causada pela preparação superficial da amostra, é desprezível, ou inferior a $2 \%$.
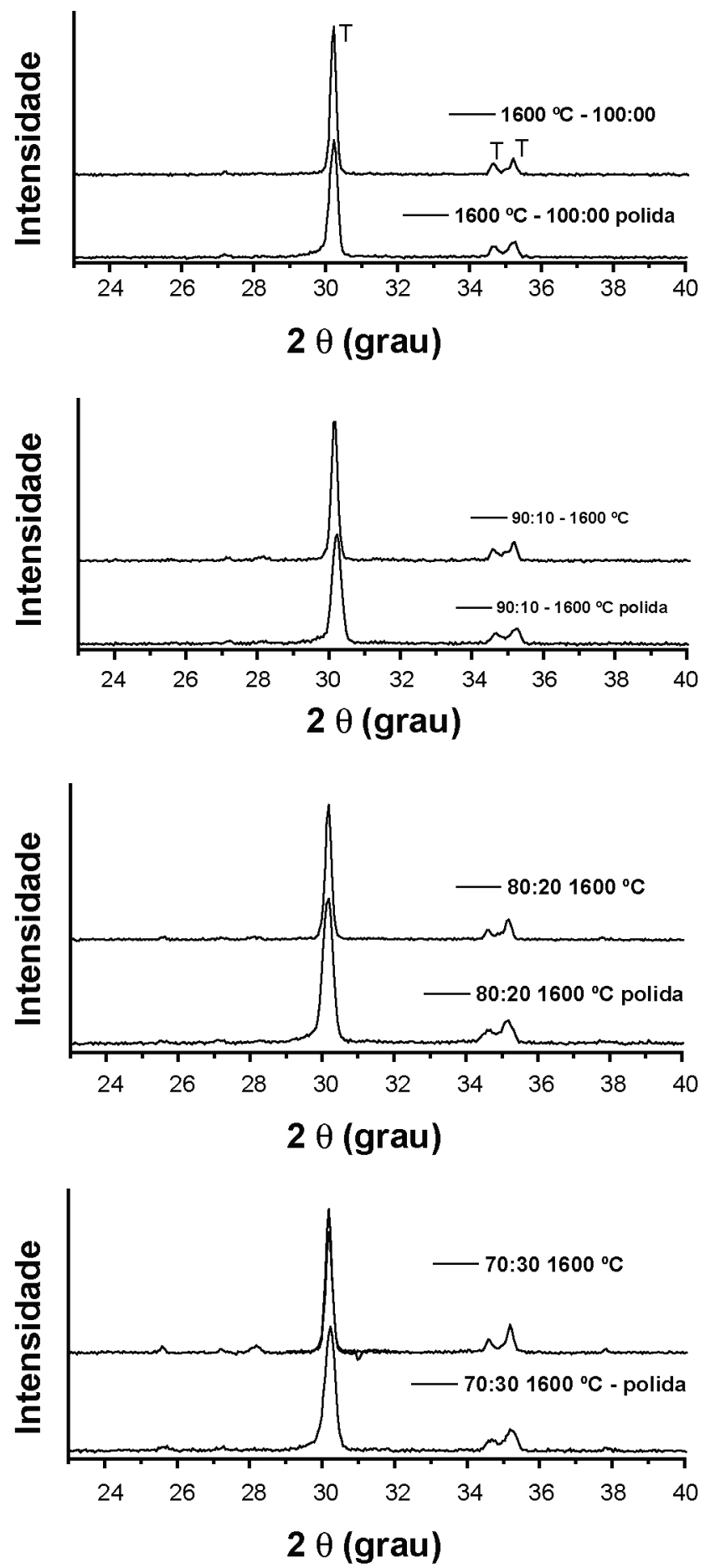

Figura 4: Difratogramas de raios $\mathrm{X}$ da superfície polida das amostras sinterizadas a $1600{ }^{\circ} \mathrm{C}$ com diferentes teores de $\mathrm{Al}_{2} \mathrm{O}_{3}$. [Figure 4: X-Ray diffraction patterns of the polished surface of samples sintered at $1600^{\circ} \mathrm{C}$.] 


\section{Microestrutura}

A Fig. 5 apresenta microestruturas características dos compósitos sinterizados a $1600{ }^{\circ} \mathrm{C}$, para as diferentes composições de $\mathrm{ZrO}_{2}-\mathrm{Al}_{2} \mathrm{O}_{3}$. Uma avaliação dos parâmetros
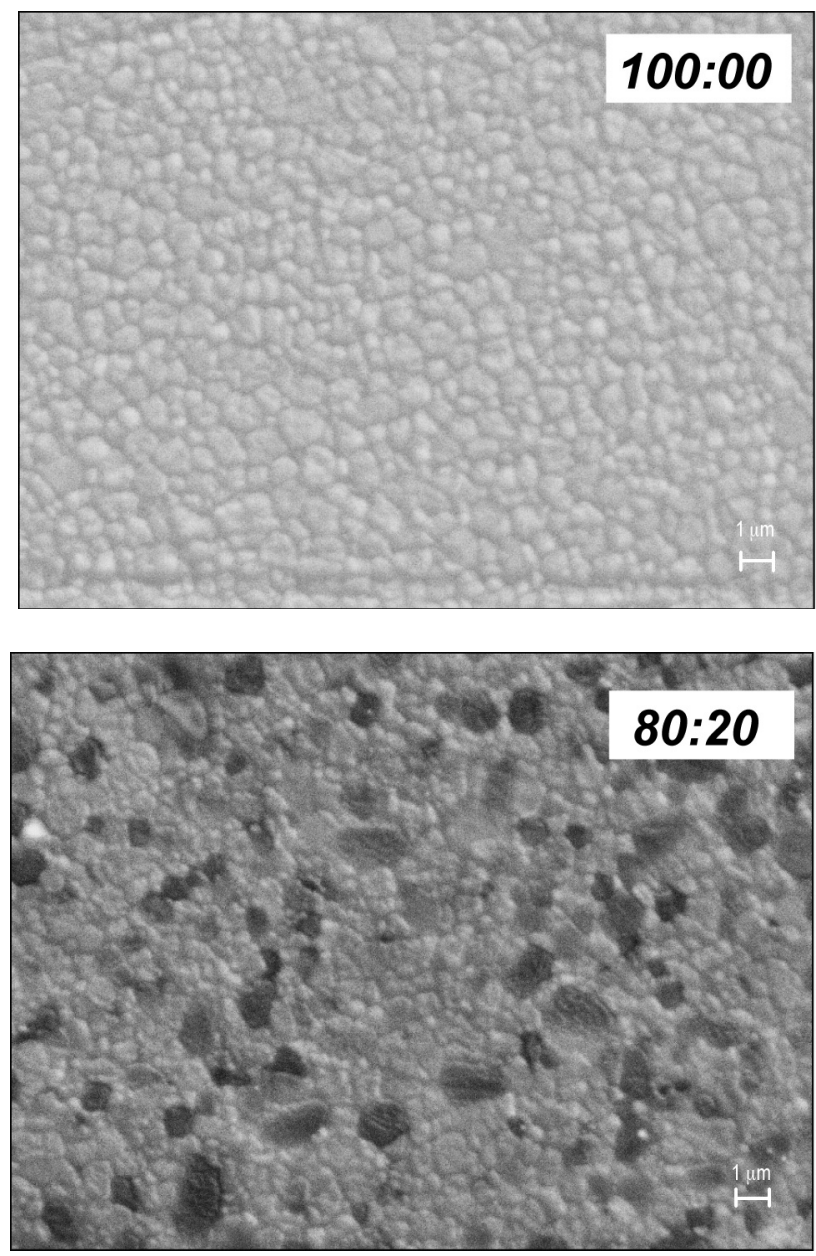

apresentaram tamanho médio de 0,5 e $1,2 \mu \mathrm{m}$, para $\mathrm{ZrO}_{2}$ e $\mathrm{Al}_{2} \mathrm{O}_{3}$, respectivamente, indicando que nas temperaturas de sinterização utilizadas nesse trabalho, a temperatura não influenciou consideravelmente no tamanho médio dos grãos de $\mathrm{ZrO}_{2}$ e $\mathrm{Al}_{2} \mathrm{O}_{3}$.
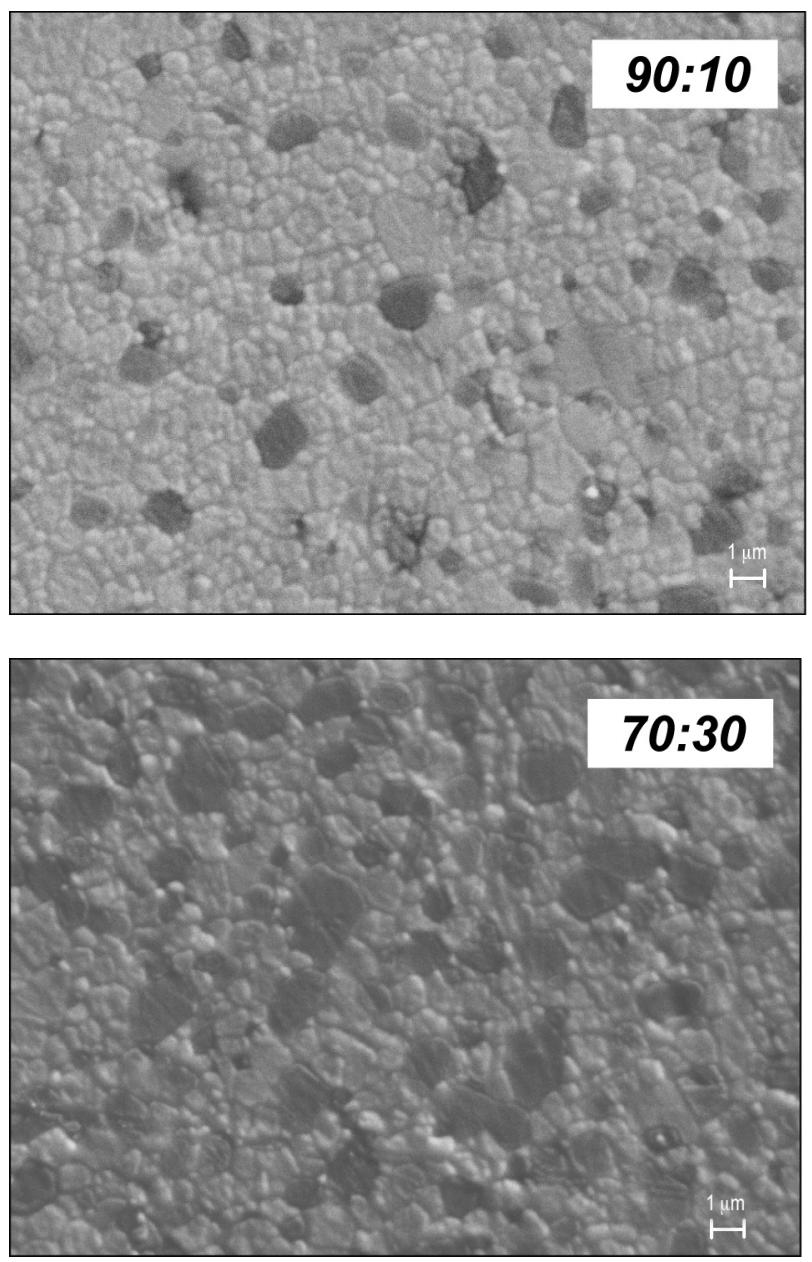

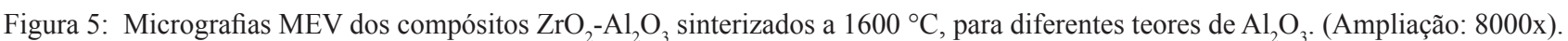
[Figure 5: SEM micrographs of the $\mathrm{ZrO}_{2}-\mathrm{Al}_{2} \mathrm{O}_{3}$ composites sintered at $1600^{\circ} \mathrm{C}$, for different $\mathrm{Al}_{2} \mathrm{O}_{3}$ content (Magnification: 8000 x). ]

microestruturais foi realizada nas amostras sinterizadas a $1600^{\circ} \mathrm{C}$, e é apresentada na Fig. 6.

É observado que os grãos de $\mathrm{ZrO}_{2}$ na matriz são da ordem de 0,4 a $0,6 \mu \mathrm{m}$, e variam discretamente em função do teor de $\mathrm{Al}_{2} \mathrm{O}_{3}$ no compósito. Em trabalhos anteriores, grãos de $\mathrm{ZrO}_{2}$ com tamanho médio da ordem de 0,3 a $0,6 \mu \mathrm{m}$ foram obtidos em compósitos sinterizados em temperaturas entre 1450 e $1600{ }^{\circ} \mathrm{C}$ [7,21]. Os grãos de $\mathrm{Al}_{2} \mathrm{O}_{3}$ apresentam um aumento considerável do tamanho médio em função do aumento de sua adição na composição de partida. Esse aumento pode ser relacionado com possível migração dos grãos de $\mathrm{Al}_{2} \mathrm{O}_{3}$ (ou íons $\mathrm{Al}^{3+}$ ) pelos contornos de grão de $\mathrm{ZrO}_{2}$ ou resultante da aglomeração das partículas durante o processamento, devido ao pequeno tamanho médio das partículas de $\mathrm{Al}_{2} \mathrm{O}_{3}$, da ordem de $0,4 \mu \mathrm{m}$. O contato entre grãos de $\mathrm{Al}_{2} \mathrm{O}_{3}$ a $1600{ }^{\circ} \mathrm{C}$ promove o crescimento dos grãos, pela redução da energia armazenada nos contornos. Por outro lado, amostras sinterizadas a $1500{ }^{\circ} \mathrm{C}, \operatorname{com} 20 \%$ de $\mathrm{Al}_{2} \mathrm{O}_{3}$

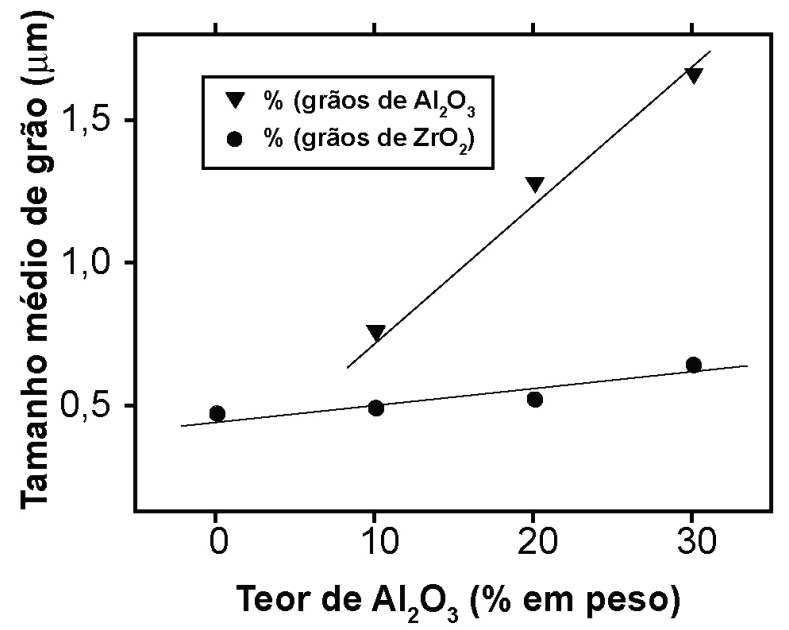

Figura 6: Tamanho médio de grãos em função do teor de $\mathrm{Al}_{2} \mathrm{O}_{3}$. [Figure 6: Average grain size as a function of the $\mathrm{Al}_{2} \mathrm{O}_{3}$ content]. 


\section{Propriedades Mecânicas}

\section{Dureza e tenacidade à fratura}

A Fig. 7 apresenta os resultados da dureza Vickers e tenacidade à fratura, $\mathrm{K}_{I C}$, das amostras, em função do teor de alumina e da temperatura de sinterização.

Pode ser observado na Fig. 1 que em todas as temperaturas utilizadas chegou-se a densidade relativa acima de 99\% DT, portanto a temperatura de sinterização não teve influência no aumento da dureza do material. Notou-se também através da Fig. 7, que a adição de alumina produz um aumento linear da dureza, alcançando valores entre 13,5 e 16,1 GPa para a adição de 0 e 30\% de alumina respectivamente, ou seja, um aumento na dureza de cerca de $20 \%$ com a adição de $30 \%$ de alumina. Pode-se notar que o aumento do teor de alumina na matriz de zircônia não reduziu a tenacidade a fratura dos compósitos, como esperado. Nesse caso, a alumina, que apresenta coeficiente de expansão térmica
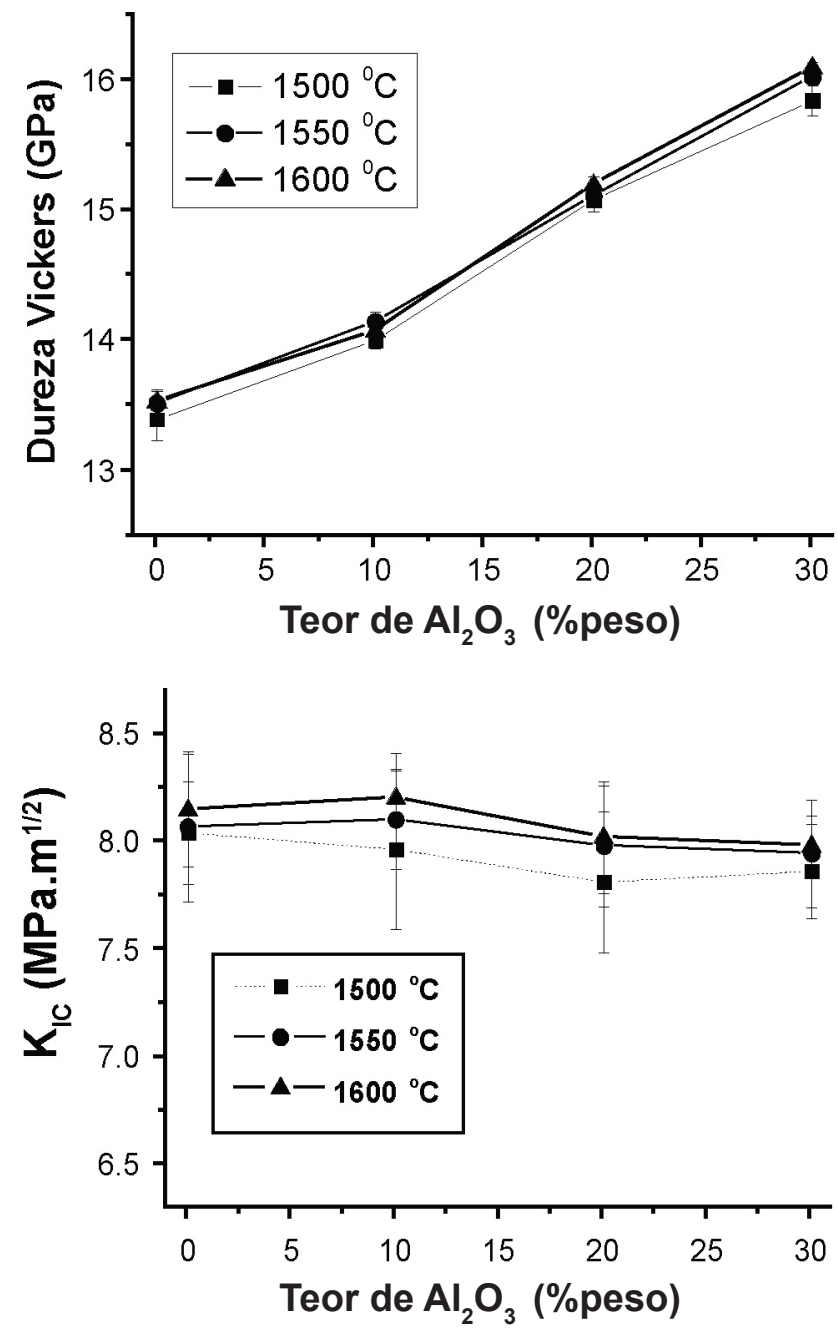

Figura 7: Influência da temperatura de sinterização e do teor de alumina na dureza e tenacidade à fratura do material sinterizado. [Figure 7: Influence of the sintering temperature and $\mathrm{Al}_{2} \mathrm{O}_{3}$ content on the hardness and fracture toughness of the sintered samples.] diferente da zircônia gera um campo de tensão ao redor de seus grãos durante o resfriamento, que resulta em barreira a propagação de trincas na matriz de zircônia. Os resultados apresentados são bastante promissores com tenacidade a fratura variando entre 7,8 e $8,2 \mathrm{MPa} \cdot \mathrm{m}^{1 / 2}$. Por outro lado, foi evidenciada a influência da tensão residual de tração na transformabilidade da $\mathrm{ZrO}_{2}$ tetragonal com um considerável efeito tenacificante nos compósitos [7]. Nesse trabalho, esse efeito positivo na tenacidade não é observado, possivelmente devido ao crescimento dos grãos de $\mathrm{ZrO}_{2}$ e principalmente de $\mathrm{Al}_{2} \mathrm{O}_{3}$ reduzindo a densidade de contornos para deflexão das trincas, um segundo possível mecanismo tenacificante desses compósitos.

Para determinar a confiabilidade desses materiais quanto a sua resistência à fratura, um lote de amostras da composição $\mathrm{ZrO}_{2}-\mathrm{Al}_{2} \mathrm{O}_{3}(80-20)$ foi submetido a ensaios de flexão em 4 pontos.

\section{Probabilidade de falha e confiabilidade das amostras}

O lote de amostras com composição 80:20(\% peso) apresentou resistência à fratura média próxima a $690 \mathrm{MPa}$.
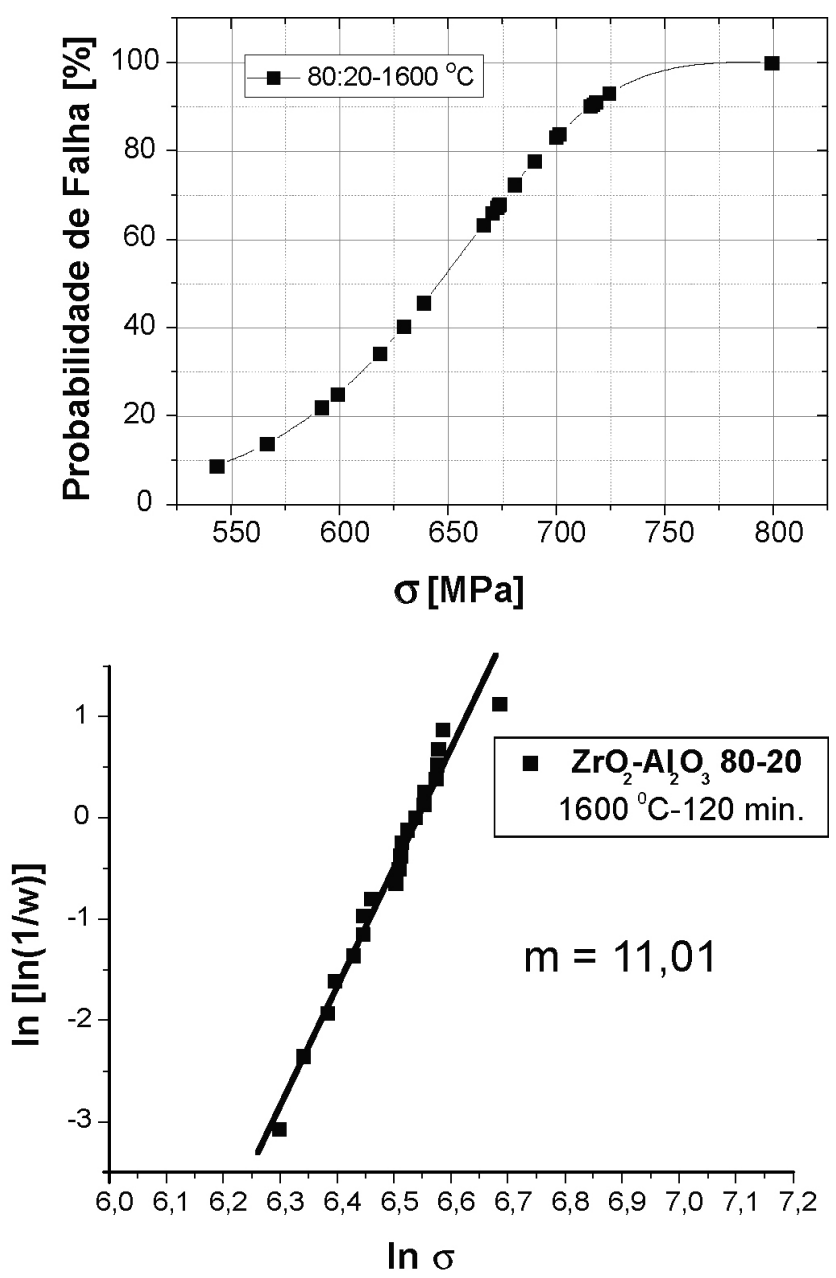

Figura 8: Probabilidade de falha e diagrama de Weibull dos compósitos $\mathrm{ZrO}_{2}: \mathrm{Al}_{2} \mathrm{O}_{3}$ sinterizados a $1600{ }^{\circ} \mathrm{C}$, por $120 \mathrm{~min}$.

[Figure 8: Weibull diagram and failure probability of the $\mathrm{ZrO}_{2}$ : $\mathrm{Al}_{2} \mathrm{O}_{3}$ composites sintered at $1600^{\circ} \mathrm{C}$, for $120 \mathrm{~min}$.] 
AFig. 8 apresenta os resultados referentes a probabilidade de falha do material, e a distribuição de Weibull das amostras de composição com $80 \%$ de $\mathrm{ZrO}_{2}$ e $20 \% \mathrm{Al}_{2} \mathrm{O}_{3}$ sinterizadas a $1600^{\circ} \mathrm{C}$ por $120 \mathrm{~min}$.

Em geral o parâmetro m, observado em uma vasta faixa de materiais cerâmicos, depende fortemente do processamento, microestrutura, distribuição de poros, e do grau de acabamento superficial. Esses valores tipicamente variam entre 3 e 15 para materiais cerâmicos [21-23], significando que materiais com $\mathrm{m}=15$ apresentam menor espalhamento de valores de resistência a fratura que materiais com $\mathrm{m}=3$. Foi reportado que lotes de materiais cerâmicos com $\mathrm{m}$ acima de 10 podem ser considerados bons e confiáveis [22].

Foi encontrado um modulo de Weibull de 11,7, para o lote analisado, demonstrando uma boa confiabilidade do lote de amostras estudadas, viabilizando sua utilização em aplicações sujeitas a esforços mecânicos, devido a sua alta confiabilidade.

\section{CONCLUSÕES}

Baseado nos resultados apresentados pode ser observado que a adição de diferentes teores de $\mathrm{Al}_{2} \mathrm{O}_{3}$ levou a um considerável aumento da dureza do compósito cerâmico de matriz de $\mathrm{ZrO}_{2}$. Nas temperaturas estudadas, a adição de $\mathrm{Al}_{2} \mathrm{O}_{3}$ na $\mathrm{ZrO}_{2}$ não influencia na densificação das amostras cerâmicas: Em todos os casos, amostras com densidade relativa acima de $99 \%$ foram obtidas. Os valores de tenacidade à fratura foram da ordem de $8 \mathrm{MPa} . \mathrm{m}^{1 / 2}$ para todas as condições e a resistência à fratura do lote analisado (amostras sinterizadas a $1600{ }^{\circ} \mathrm{C} / 120$ min) foi da ordem de $690 \mathrm{MPa}$, apresentando um módulo de Weibull próximo de 12, o que indica um lote de amostras de boa confiabilidade. Esse resumo dos resultados leva a acreditar que os compósitos desenvolvidos nesse trabalho apresentam propriedades promissoras para utilização como componentes cerâmicos para sistemas de implantes.

\section{AGRADECIMENTOS}

Os autores gostariam de agradecer a FAPESP (Proc. 04/04386-1) pelo apoio financeiro dado a esse projeto.

\section{REFERÊNCIAS}

[1] L. L. Hench, Bioceramics, J. Am. Ceram. Soc. 81, 7 (1998) 1705-1728.

[2] D. F. Willians, Biofuncionality and biocompatibility, Medical and Dental Materials, New York (1992).

[3] L. L. Hench, J Wilson, An Introduction to Bioceramics, Advanced Series in Ceramics, 1, World Scientific, Singapura (1993) pp.1-23.

[4] A. H. De Aza, J. Chevalier, G. Fantozzi, Crack grouth resistance of alumina, zirconia and zirconia toughened alumina ceramics for joint prostheses, Biomaterials $\mathbf{2 3}$ (2002) 937-945.

[5] R. Stevens, An introduction to zirconia: Zirconia and zirconia ceramics, $2^{\text {nd }}$ Ed., Magnesium Elektrum Publ. n. 113 (1986).
[6] D. Basu, B. K. Sarkar, Toughness determination of zirconia toughened alumina ceramics from growth of indentation-induced cracks, J. Mater. Res. 11, 12 (1996) 3057-3062.

[7] B. Basu, J. Vleugels, O. Van Der Biest, $\mathrm{ZrO}_{2}-\mathrm{Al}_{2} \mathrm{O}_{3}$ composites with tailored toughness, J Alloys and Comp. 372, 1-2 (2004) 278-284.

[8] C. Piconi, G. Maccauro, Zirconia as a ceramic biomaterial, Biomaterials 20 (1999) 1-25.

[9] C. Piconi, W. Burger, H. G. Richter, Y-TZP ceramics for artificial joint replacements. Biomaterials 19 (1998) 1489-1494.

[10] R. Stevens, Zirconia: second phase particle transformation toughening of ceramics, Trans. Brit. Ceram. Soc. 80 (1981) 81-85.

[11] M. Rühle, A. Stecker, D. Waidelich, B. Kraus, In situ observations of stress-induced phase transformation in $\mathrm{ZrO}_{2}$ containing ceramics, in Eds. N. Claussen, M. Rülle, A. H. Heuer, Advances in Ceramics, Science and Technology of Zirconia II, The American Ceramic Society (1984) 256-274.

[12] B. L. Karihaloo, Contributions of $t-m$ phase transformation to the toughening of ZTA, J. Am. Ceram. Soc. 74 (1991) 1703-1706.

[13] P. F. Becher, K. B. Alexander, W. Warmick, Influence of $\mathrm{ZrO}_{2}$ grain size and content on the transformation response in the $\mathrm{Al}_{2} \mathrm{O}_{3}-\mathrm{ZrO}_{2}\left(12 \% \mathrm{~mol} \mathrm{CeO}_{2}\right)$ system, J. Am. Ceram. Soc. 76 (1993), 657-663.

[14] G. Gregori, W. Burger, V. Sergo, Piezo-spectroscopic analysis of the residual stress in zirconia-toughened alumina ceramics: the influence of the tetragonal-to-monoclinic transformation, Mater. Sci. Eng. A 271 (1999) 401-406.

[15] R. C. Garvie, P. S. Nicholson, Phase analysis in zirconia systems, J. Am. Ceram. Soc. 55 (1972) 303-305.

[16] H. Toraya, M. Yoshimura, S. Somiya, Calibration curve for quantitative analysis of the monoclinic tetragonal $\mathrm{ZrO}_{2}$ system by X-ray diffraction, J. Am. Ceram. Soc. 67 (1984) 119-121.

[17] K. Niihara, R. Moreno, D. P. H. Hasselman, Evaluation of $\mathrm{K}_{\mathrm{IC}}$ of brittle solids by the indentation method with low crack-to-indent ratios, J. Mater. Sci. Lett. 1 (1982) 13-16.

[18] W. Weibull, A statistical distribution function of wide applicability, J. Appl. Mechanics (1958) 293-297.

[19] Normennausschuss Materialprüfung im DIN Deutsches Institut für Normung e.V. 4-Punkt- Biegeversuch, Statistische Auswertung, Ermittlung der Weibull-Parameter (1993). DIN 51 110, Teil 3.

[20] L. Ruiz, M. J. Readey, Effect of heat treatment on grain size, phase assemblage, and mechanical properties of $3 \mathrm{~mol} \%$ Y-TZP , J. Am. Ceram. Soc. 79, 9 (1996) 2331-2340.

[21] W. J. Walker, J. S., Reed, J. Am. Ceram. Soc. 82, 1 (1996) 50-56.

[22] G. D. Quinn, Strenght and Proof Testing, Engineered Materials Handbook, vol. 4, Ceramics and Glasses. ASM International, Metals Park, OH (1991) 585-598.

[23] E. D. Zanotto, A. R. Migliore Jr., Cerâmica 37, 247 (1991) 07-16.

(Rec. 27/01/2006, Rev. 13/11/2006, 19/12/2006, Ac. 22/02/2007) 